\title{
Article \\ Global Well-Posedness and Analyticity of the Primitive Equations of Geophysics in Variable Exponent Fourier-Besov Spaces
}

\author{
Muhammad Zainul Abidin 1,*(D), Naeem Ullah ${ }^{2}$ and Omer Abdalrhman Omer 1,*(D) \\ 1 College of Mathematics and Computer Science, Zhejiang Normal University, Jinhua 321004, China \\ 2 Department of Mathematics, Islamia College, Peshawar 25000, Pakistan; naeemullahstd@icp.edu.pk \\ * Correspondence: mzainulabidin@zjnu.edu.cn (M.Z.A.); omeraomer@zjnu.edu.cn (O.A.O.)
}

Citation: Abidin, M.Z.; Ullah, N.; Omer, O.A. Global Well-Posedness and Analyticity of the Primitive Equations of Geophysics in Variable Exponent Fourier-Besov Spaces. Symmetry 2022, 14, 165. https:// doi.org/10.3390/sym14010165

Academic Editors: Yagub Sharifov and Nazim Mahmudov

Received: 13 December 2021

Accepted: 10 January 2022

Published: 14 January 2022

Publisher's Note: MDPI stays neutral with regard to jurisdictional claims in published maps and institutional affiliations.

Copyright: (c) 2022 by the authors. Licensee MDPI, Basel, Switzerland. This article is an open access article distributed under the terms and conditions of the Creative Commons Attribution (CC BY) license (https:// creativecommons.org/licenses/by/ $4.0 /)$.

\begin{abstract}
We consider the Cauchy problem of the three-dimensional primitive equations of geophysics. By using the Littlewood-Paley decomposition theory and Fourier localization technique, we prove the global well-posedness for the Cauchy problem with the Prandtl number $P=1$ in variable exponent Fourier-Besov spaces for small initial data in these spaces. In addition, we prove the Gevrey class regularity of the solution. For the primitive equations of geophysics, our results can be considered as a symmetry in variable exponent Fourier-Besov spaces.
\end{abstract}

Keywords: well-posedness; primitive equations; analyticity; variable exponent Fourier-Besov spaces

\section{Introduction}

The primitive equations of geophysics are a fundamental mathematical model that describes the dynamics of the oceans and atmosphere on a large-scale. For more study of these equations and their applications, see the monographs [1-3]. The vast atmospheric and oceanic circulations, as geophysical flows, apart from obeying the fundamental rules of fluid mechanics, are also influenced by the essential properties of the Earth. The effects of Earth's rotation and the stratification of density caused by gravity are the main properties that differentiate fluid flow in the oceans and atmosphere.

The rotation produces the Coriolis force, which mathematically results in the occurrence of the Poincare wave. Waves generated by Poincare are dispersive and travel rapidly inside the region. Additionally, the Coriolis effect generates fluid vertical rigidity, which is explained in the Taylor-Proudman theorem. The theorem states that when a fluid rotates fast, all particles on the same vertical have the same horizontal velocity.

Gravity has a tendency to pull the denser fluid downwards and the light fluid up in the same direction. The fluid has a steady stratified structure under equilibrium conditions and consists of horizontal layers that are stacked vertically. Gravity makes systematic attempts to restore equilibrium as fluid motions disrupt it. The Brunt-Vaisala frequency and buoyancy are used to determine the importance of these stratifications. Small perturbations result in the generation of additional dispersive waves known as internal gravity waves. In geophysical fluids, an interesting situation occurs when there is a significant interaction between rotation and stratification, but neither is dominating.

On this basis, the large motions of the oceans and atmosphere are generally formulated by the following $3 \mathrm{D}$ primitive equations:

$$
\begin{cases}a_{t}+\mu \Delta a+(a \cdot \nabla) a+\Omega e_{3} \times a+\nabla p=\omega \vartheta e_{3} & (t, y) \in(0, \infty) \times \mathbb{R}^{3}, \\ \vartheta_{t}+v \Delta \vartheta+(a \cdot \nabla) \vartheta=-M^{2} a_{3} & (t, y) \in(0, \infty) \times \mathbb{R}^{3}, \\ \operatorname{div} a=0 & (t, y) \in(0, \infty) \times \mathbb{R}^{3},\end{cases}
$$


where $a$ and $p$ denote the unknown velocity field and pressure of the fluid. A scalar function $\vartheta$ represents the density fluctuation in the fluid, while $a_{0}$ is the given initial velocity field that satisfies $\nabla \cdot a=0 . \omega, \mu$ and $v$ are positive constants related to gravity, viscosity and diffusivity, respectively. The Coriolis parameter $\Omega \in \mathbb{R}$ denotes the speed of rotation around the vertical unit vector $e_{3}=(0,0,1) . M \geq 0$ is the stratification parameter represents the Brunt-Visala wave frequency. The ratios $P:=\frac{\mu}{v}$ and $B:=\frac{\Omega}{M}$ represents the Prandtl number and the "Burgers"number of geophysics, respectively. The derivation of the equations and more explanations related to their physical foundation can be found in $[4,5]$ and the references therein.

If $M=0, \Omega=0$ and $\vartheta=0$, Equation (1) is reduced to the following 3D incompressible Navier-Stokes (NS) equations

$$
\begin{cases}a_{t}+\mu \Delta a+(a \cdot \nabla) a+\nabla p=0 & (t, y) \in(0, \infty) \times \mathbb{R}^{3}, \\ \operatorname{div} a=0 & (t, y) \in(0, \infty) \times \mathbb{R}^{3} .\end{cases}
$$

The mathematical theory of Equation (2) comes from the well-known article by Jean Leray [6], which studies the global existence of NS equations with respect to weak solutions, but the uniqueness and regularity question are still open in three dimensions (3D). The first well-posedness result of Equation (2) was obtained by Fujita and Kato [7], which shows the local well-posedness when the $H^{\beta}\left(\mathbb{R}^{3}\right)$ with $\beta \geq \frac{1}{2}$ and global well-posedness belonging to $H^{\frac{1}{2}}\left(\mathbb{R}^{3}\right)$ when the initial data are small. This solution was further extended to a family of function spaces. For a detailed study in this direction, see [8-10]. It is important to note that results related to well-posedness are proved in $\dot{\mathcal{B}}_{\rho, r}^{-1+\frac{3}{\rho}}\left(\mathbb{R}^{3}\right)$ for $1 \leq r \leq \infty, 1 \leq \rho<\infty$ for $1 \leq r \leq \infty, 1 \leq \rho<\infty$ by Cannone et al. [8], Koch and Tataru [9] in $B M O^{-1}$ and Lei [10]. However, Bourgain and Pavlović [11] studied the ill-posedness of Equation (2) in $\dot{\mathcal{B}}_{q}^{-1, \infty}\left(\mathbb{R}^{3}\right)$ for $1 \leq q \leq \infty$ and Yoneda [12] in $\dot{F}_{\infty, r}^{-1}\left(\mathbb{R}^{3}\right)$ for $2<r \leq \infty$.

If $\Omega \neq 0, M=0$ and $\vartheta=0$, Equation (1) reduces to 3D incompressible NS equations with Coriolis force

$$
\begin{cases}a_{t}+\mu \Delta a+(a \cdot \nabla) a+\Omega e_{3} \times a+\nabla p=0 & (t, y) \in(0, \infty) \times \mathbb{R}^{3}, \\ \text { diva }=0 & (t, y) \in(0, \infty) \times \mathbb{R}^{3} .\end{cases}
$$

The global well-posedness of Equation (3) has also received a great interest, for more study in this direction, see [4,13]. In particular, Giga et al. [14] proved the global wellposedness of Equation (3) for small initial data in $F M_{0}^{-1}\left(\mathbb{R}^{3}\right)$, Hieber and Shibata [15] in $H^{\frac{1}{2}}\left(\mathbb{R}^{3}\right)$, Fang et al. [16] in $\mathcal{F} \dot{\mathcal{B}}_{\rho, r}^{2-\frac{3}{\rho}}\left(\mathbb{R}^{3}\right)$ when $1 \leq r<\infty$ and $1<\rho \leq \infty$, Konieczny and Yoneda [17] in $\mathcal{F} \dot{\mathcal{B}}_{1,1}^{-1}\left(\mathbb{R}^{3}\right) \cap \mathcal{F} \dot{\mathcal{B}}_{1,1}^{0}\left(\mathbb{R}^{3}\right)$, Iwabuchi and Takada [18] and in $\mathcal{F} \dot{\mathcal{B}}_{1,2}^{-1}\left(\mathbb{R}^{3}\right)$.

In this paper, we consider the Cauchy problem for $3 \mathrm{D}$ primitive equations of geophysics.

$$
\begin{cases}a_{t}+\mu \Delta a+(a \cdot \nabla) a+\Omega e_{3} \times a+\nabla p=g \vartheta e_{3} & (t, y) \in(0, \infty) \times \mathbb{R}^{3}, \\ \vartheta_{t}+v \Delta \vartheta+(a \cdot \nabla) \vartheta=-M^{2} v_{3} & (t, y) \in(0, \infty) \times \mathbb{R}^{3}, \\ \operatorname{div} a=0, & (t, y) \in(0, \infty) \times \mathbb{R}^{3}, \\ a(y, 0)=a_{0}, \vartheta(y, 0)=\vartheta_{0} & y \in \mathbb{R}^{3},\end{cases}
$$

where $v_{0}$ and $\theta_{0}$ are the given initial data.

The following is a brief review of the existence of solutions to Equation (4). Chemin [19] established the global well-posedness of Equation (4) using very careful energy calculations for initial data belonging to $\dot{H}^{1}\left(\mathbb{R}^{3}\right) \cap \dot{H}^{1}\left(\mathbb{R}^{3}\right)$, assuming that $\Omega=M^{2}$ is sufficiently large. Babin et al. [4] obtained the global solvability of Equation (4) in $H^{\beta}\left(\mathbb{T}^{3}\right)$ with $\beta \geq \frac{3}{4}$ for small initial data and large enough $M$. Koba et al. [20] obtained the global well-posedness to Equation (4) by considering $P=1$ in $\dot{H}^{\frac{1}{2}}\left(\mathbb{R}^{3}\right) \cap \dot{H}^{1}\left(\mathbb{R}^{3}\right)$. Additionally, they established the global existence to the solution of Equation (4), when the initial data are small and belonging 
to $\dot{H}^{\frac{1}{2}}\left(\mathbb{R}^{3}\right)$. Iwabuchi et al. [21] recently proved the well-posedness to Equation (4) with $P=1$ for the large oscillatory component of initial data belonging to $\dot{H}^{\beta}\left(\mathbb{R}^{3}\right)\left(\frac{1}{2}<\beta \leq \frac{5}{8}\right)$ and the small quasi-geostrophic component belonging $\dot{H}^{\frac{1}{2}}\left(\mathbb{R}^{3}\right)$ with sufficiently large $M$, by utilizing the full dispersion effect of stratification and rotation. When the rotation is fast, Charve and Ngo [22] proved the global well-posedness for primitive equations. Recently, Abbassi et al. [23] obtained the local well-posedness and global well-posedness results of Equation (4) for small initial data belonging to Fourier-Besov-Morrey spaces. We can conclude from the above-mentioned results that there is a significant difference in the wellposedness of Equation (4) and the incompressible NS Equation (2) or the incompressible NS equations with Coriolis force (3). Motivated by [17,18,22], we can extend the well-posedness results of Equations (2) or (3) to the Equation (4).

In this paper, the global well-posedness of the Equation (4) is considered in variable exponent Fourier-Besov space $\mathcal{F} \dot{\mathcal{B}}_{\rho(\cdot), q}^{\beta(\cdot)}$. As these spaces are symmetric, the results proved in this work also have the property of symmetry. The space of variable integrability, also known as Lebesgue space $L^{\rho(\cdot)}\left(\mathbb{R}^{n}\right)$, originated with the article of Orlicz [24], and was further studied by Musielak [25] and Nakano [26]; however, the present development started with the papers of Kovaik and Rakosnik [27]. It has received a great deal of attention in harmonic analysis, as evidenced by $[28,29]$. For more study in this direction, we refer the readers to [30] and the references therein. In addition to the theoretical study, these function spaces have significant uses in image processing [31], fluid dynamics [32], and partial differential equations [33].

Throughout the paper, we write $f \lesssim g$ to represent $f \leq C g$, where $C$ is a positive constant. In Section 2, we give definitions of some important function spaces and some useful propositions related to variable exponent function spaces. In Section 3, we establish the global well-posedness result. In Section 4, the analyticity of global solution is obtained.

\section{Preliminaries}

First, we give an overview of some important harmonic analysis related to the variable exponent function spaces.

Definition 1. For the measurable function $\rho(\cdot)$, let

$$
\mathcal{P}_{0}:=\left\{\rho(\cdot): \mathbb{R}^{n} \rightarrow(0, \infty) ; 0<p_{-}=\underset{y \in \mathbb{R}^{n}}{\operatorname{esssup}} \rho(y), \underset{y \in \mathbb{R}^{n}}{\operatorname{esssup}} \rho(y)=p_{+}<\infty\right\} .
$$

The variable exponent Lebesgue space is:

$$
L^{\rho(\cdot)}\left(\mathbb{R}^{n}\right)=\left\{a: \mathbb{R}^{n} \rightarrow \mathbb{R} \text { is measurable, } \int_{\mathbb{R}^{n}}|a(y)|^{\rho(y)} d x<\infty\right\}
$$

with norm

$$
\|a\|_{L^{\rho(\cdot)}}=\inf \left\{\alpha>0: \int_{\mathbb{R}^{n}}\left(\frac{|a(y)|}{\alpha}\right)^{\rho(y)} d x \leq 1\right\} .
$$

To distinguish between constant and variable exponents, we use the notation $\rho$ for constant exponents and $\rho(\cdot)$ for variable exponents. In addition, $\left(L^{\rho(\cdot)}\left(\mathbb{R}^{n}\right),\|a\|_{L^{\rho(\cdot)}}\right)$ is a Banach space.

Definition 2. Let $\rho(\cdot), q(\cdot) \in \mathcal{P}_{0}\left(\mathbb{R}^{n}\right)$, the mixed variable exponent Lebesgue-sequence space is the set of all sequences $\left\{a_{j}\right\}_{j \in \mathbb{Z}}$ of measurable functions in $\mathbb{R}^{n}$ such that

$$
\left\|\{a\}_{j \in \mathbb{Z}}\right\|_{l^{q(\cdot)}\left(L^{\rho(\cdot)}\right)}:=\inf \left\{\lambda>0, \varrho_{l^{q(\cdot)}\left(L^{\rho(\cdot)}\right)}\left(\left\{\frac{a_{j}}{\lambda}\right\}_{j \in \mathbb{Z}}\right) \leq 1\right\}<\infty,
$$


where

$$
\varrho_{q^{g(\cdot)}\left(L^{\rho(\cdot)}\right)}\left(\{a\}_{j \in \mathbb{Z}}\right):=\sum_{i \in Z} \inf \left\{\lambda_{j}>0, \int_{\mathbb{R}^{n}}\left(\left\{\frac{a_{j}(y)}{\lambda_{j}^{\frac{1}{g(y)}}}\right\}\right)^{\rho(y)} \leq 1\right\} .
$$

Note that, if $q_{+}<\infty$, then $\varrho_{l^{q(\cdot)}\left(L^{\rho(\cdot)}\right)}\left(\{a\}_{j \in \mathbb{Z})}\right):=\sum_{j \in \mathbb{Z}}\left\|\left.a_{j}\right|^{q(\cdot)}\right\|_{L^{\frac{\rho(\cdot)}{q(\cdot)}}}$.

The $L^{\rho(\cdot)}$ does not have the same desired properties like $L^{\rho}$ as it is not translation invariant. To ensure the boundedness of Hardy-Littlewood maximal operator on $L^{\rho(\cdot)}\left(\mathbb{R}^{n}\right)$, it is assumed that the standard conditions given below are satisfied.

(i) Locally log-Hölder continuous: For any $y, x \in \mathbb{R}^{n}$, there exists a constant $C_{\log }(\rho)$ such that

$$
|\rho(y)-\rho(x)| \leq \frac{C_{\log }(\rho)}{\log \left(e+|y-x|^{-1}\right)}, x \neq y ;
$$

(ii) Globally log-Hölder continuous: For all $y \in \mathbb{R}^{n}$, there exists a constant independent of $y$ and some other constant $C_{\log }(\rho)$ such that

$$
\left|\rho(y)-p_{\infty}\right| \leq \frac{C_{\log }(\rho)}{\log (e+|y|)} .
$$

The set of all functions $\rho(\cdot): \mathbb{R}^{n} \rightarrow \mathbb{R}$ satisfies (i) and (ii) is denoted by $C^{\log }\left(\mathbb{R}^{n}\right)$.

Definition 3. Let $\beta(\cdot) \in C^{\log }\left(\mathbb{R}^{n}\right)$ and $\rho(\cdot), q(\cdot) \in \mathcal{P}_{0}\left(\mathbb{R}^{n}\right) \cap C^{\log }\left(\mathbb{R}^{n}\right)$. The variable exponent homogeneous Besov space $\dot{\mathcal{B}}_{\rho(\cdot), q(\cdot)}^{\beta(\cdot)}$ is

$$
\dot{\mathcal{B}}_{\rho(\cdot), q(\cdot)}^{\beta(\cdot)}=\left\{a \in \mathcal{D}^{\prime}\left(\mathbb{R}^{n}\right) ; \quad\|a\|_{\mathcal{B}_{\rho(\cdot), q(\cdot)}^{\beta(\cdot)}}<\infty\right\}
$$

with norm

$$
\|a\|_{\mathcal{B}_{\rho(\cdot), q(\cdot)}^{\beta(\cdot)}}=\left\|\left\{2^{j s(\cdot)} \dot{\Delta}_{j} a\right\}_{j \in \mathbb{Z}}\right\|_{\varrho_{1 q(\cdot)\left(L^{\rho(\cdot)}\right)}} .
$$

The space $\mathcal{D}^{\prime}\left(\mathbb{R}^{n}\right)$ represents the dual space of

$$
\mathcal{D}\left(\mathbb{R}^{n}\right)=\left\{a \in \mathscr{T}\left(\mathbb{R}^{n}\right):\left(D^{\alpha} a\right)(0)=0, \forall \alpha\right\} .
$$

Definition 4. Let $\beta(\cdot) \in C^{\log }\left(\mathbb{R}^{n}\right)$ and $\rho(\cdot), q(\cdot) \in \mathcal{P}_{0}\left(\mathbb{R}^{n}\right) \cap C^{\log }\left(\mathbb{R}^{n}\right)$. The homogeneous variable exponent Fourier-Besov space $\mathcal{F} \dot{\mathcal{B}}_{\rho(\cdot), q(\cdot)}^{\beta(\cdot)}$ is defined by

$$
\mathcal{F} \dot{\mathcal{B}}_{\rho(\cdot), q(\cdot)}^{\beta(\cdot)}=\left\{a \in \mathcal{D}^{\prime}\left(\mathbb{R}^{n}\right) ; \quad\|a\|_{\mathcal{F} \dot{\mathcal{B}}_{\rho(\cdot), q(\cdot)}^{\beta(\cdot)}}<\infty\right\}
$$

with norm

$$
\|a\|_{\mathcal{F} \dot{B}_{\rho(\cdot), q(\cdot)}^{\beta(\cdot)}}=\left\|\left\{2^{j s(\cdot)} \dot{\Delta}_{i} a\right\}_{j \in \mathbb{Z}}\right\|_{\varrho_{l q(\cdot)\left(L^{\rho(\cdot)}\right)}}
$$

Definition 5. For $1 \leq r, \gamma \leq \infty$ and $T \in(0, T]$, the variable exponent of Chemin-Lerner type in a homogeneous Fourier-Besov space $\mathcal{L}^{\gamma}\left(0, T ; \mathcal{F} \dot{\mathcal{B}}_{\rho(\cdot), r}^{\beta(\cdot)}\right)$ is defined by

$$
\mathcal{L}^{\gamma}\left(0, T ; \mathcal{F} \dot{\mathcal{B}}_{\rho(\cdot), r}^{\beta(\cdot)}\right)=\left\{a \in \mathcal{D}^{\prime}\left(\mathbb{R}^{n}\right) ; \quad\|a\|_{\mathcal{L}^{\gamma}\left(0, T ; \mathcal{F} \dot{\mathcal{B}}_{\rho(\cdot), r}^{\beta(\cdot)}\right)}<\infty\right\}
$$


with norm

$$
\|a\|_{\mathcal{L} \gamma\left(0, T ; \mathcal{F} \dot{\mathcal{B}}_{\rho(\cdot), r}^{\beta(\cdot)}\right)}=\left(\sum_{j \in \mathbb{Z}}\left\|2^{j s(\cdot)} \dot{\Delta}_{j} a\right\|_{L_{T}^{\gamma} L^{\rho(\cdot)}}^{r}\right)^{\frac{1}{r}} .
$$

Proposition 1. The following estimates hold for variable exponent function spaces.

(1) Hölder's inequality[29]. Let $\mathcal{P}(\mathbb{R})^{n}$ is the set of all measurable functions $\rho: \mathbb{R}^{n} \rightarrow[1, \infty)$ and $\rho(\cdot), q(\cdot), r(\cdot) \in \mathcal{P}(\mathbb{R})^{n}$ such that $\underset{y \in \mathbb{R}^{n}}{\operatorname{esssup}} \rho(y)=p_{+}<\infty$ and $\frac{1}{r(y)}=\frac{1}{\rho(y)}+\frac{1}{q(y)}$. Then,

$$
\|a b\|_{L^{r(\cdot)\left(\mathbb{R}^{n}\right)}}=\|a\|_{L^{\rho(\cdot)}\left(\mathbb{R}^{n}\right)}\|b\|_{L^{q(\cdot)\left(\mathbb{R}^{n}\right)}} .
$$

(2) Sobolev Embedding [34]. Let $s_{0}(\cdot), s_{1}(\cdot) \in L^{\infty}$ with $s_{0}(\cdot) \geq s_{1}(\cdot)$ and $p_{0}(\cdot), p_{1}(\cdot), q(\cdot) \in$ $\mathcal{P}\left(\mathbb{R}^{n}\right)$. If $\frac{1}{q(\cdot)}$ and $s_{0}(\cdot)-\frac{n}{p_{0}(\cdot)}=s_{1}(\cdot)-\frac{n}{p_{1}(\cdot)}$ are Locally log-Hölder continuous, then

$$
\dot{\mathcal{B}}_{p_{0}(\cdot), q(\cdot)}^{s_{0}(\cdot)} \hookrightarrow \dot{\mathcal{B}}_{p_{1}(\cdot), q(\cdot)}^{s_{1}(\cdot)}
$$

(3) ([34]) Let $s_{0}(\cdot), s_{1}(\cdot) \in L^{\infty}$ with $s_{0}(\cdot) \geq s_{1}(\cdot)$ and $p_{0}(\cdot), p_{1}(\cdot), q(\cdot) \in \mathcal{P}\left(\mathbb{R}^{n}\right)$. If $\frac{1}{q_{0}(\cdot)}, \frac{1}{q_{1}(\cdot)}$ and $s_{0}(\cdot)-\frac{n}{p_{0}(\cdot)}=s_{1}(\cdot)-\frac{n}{p_{1}(\cdot)}+\epsilon(y)$ are Locally log-Hölder continuous, where esssup $\epsilon>0$, then

$$
\dot{\mathcal{B}}_{p_{0}(\cdot), q_{0}(\cdot)}^{s_{0}(\cdot)} \hookrightarrow \dot{\mathcal{B}}_{p_{1}(\cdot), q_{1}(\cdot)}^{s_{1}(\cdot)}
$$

Proposition 2. ([35]) Let $\beta>0,1 \leq \gamma, \gamma_{1}, \gamma_{2}, \rho, q, r, \sigma \leq \infty, \frac{1}{\sigma}=\frac{1}{\rho}+\frac{1}{r}$ and $\frac{1}{\gamma}=\frac{1}{\gamma_{1}}+\frac{1}{\gamma_{2}}$. Then,

$$
\|a b\|_{\mathcal{L}^{\gamma} \dot{\mathcal{B}}_{\sigma, q}^{\beta}} \lesssim\|a\|_{\mathcal{L}^{\gamma_{1} \dot{\mathcal{B}}_{\rho, q}^{\beta}}}\|b\|_{\mathcal{L}^{\gamma_{2} L^{r}}}+\|b\|_{\mathcal{L}^{\gamma_{1} \dot{\mathcal{B}}_{\rho, q}^{\beta}}}\|a\|_{\mathcal{L}^{\gamma_{2} L^{r}}}
$$

\section{The Well-Posedness}

In this section, we first convert Equation (4) to an equivalent integral form. We refer the readers to [20] for a comprehensive derivation of the equivalent integral form.

By setting

$\mathcal{M}:=M \sqrt{\omega}, u:=\left(u^{1}, u^{2}, u^{3}, u^{4}\right):=\left(a^{1}, a^{2}, a^{3}, \sqrt{\omega} \vartheta / M\right), u_{0}:=\left(u_{0}^{1}, u_{0}^{2}, u_{0}^{3}, u_{0}^{4}\right):=$ $\left(a_{0}^{1}, a_{0}^{2}, a_{0}^{3}, \sqrt{\omega} \theta_{0} / M\right)$ and $\tilde{\nabla}:=\left(\partial_{1}, \partial_{2}, \partial_{3}, 0\right)$, Equation (4) can be written as follows:

$$
\begin{cases}u_{t}+\mathscr{A} u+\mathscr{B} u+\tilde{\nabla} p=-(u \cdot \tilde{\nabla}) & (t, y) \in(0, \infty) \times \mathbb{R}^{3}, \\ \tilde{\nabla} \cdot u=0, & (t, y) \in(0, \infty) \times \mathbb{R}^{3}, \\ u(y, 0)=u_{0} & y \in \mathbb{R}^{3},\end{cases}
$$

where

$$
\mathscr{A}:=\left(\begin{array}{cccc}
-\mu \Delta & 0 & 0 & 0 \\
0 & -\mu \Delta & 0 & 0 \\
0 & 0 & -\mu \Delta & 0 \\
0 & 0 & 0 & -\mu \Delta
\end{array}\right), \mathscr{B}:=\left(\begin{array}{cccc}
0 & -\Omega & 0 & 0 \\
\Omega & 0 & 0 & 0 \\
0 & 0 & 0 & -\mathcal{M} \\
0 & 0 & \mathcal{M} & 0
\end{array}\right) .
$$

In order to solve Equation (4), we consider the following integral equation

$$
u(t)=S_{\Omega, \mathcal{M}}(t) u_{0}+\int_{0}^{t} S_{\Omega, \mathcal{M}}(t-\mathcal{T}) \mathbb{P} \tilde{\nabla} \cdot(u \otimes u) d \mathcal{T}
$$

where the Helmholtz projection onto the divergence-free vector fields $\tilde{\mathbb{P}}:=\left(\tilde{\mathbb{P}}_{m n}\right)_{4 \times 4}$ is given by

$$
\tilde{\mathbb{P}}_{m n}= \begin{cases}\delta_{m n}+R_{m} R_{n} & 1 \leq m, n \leq 3 \\ \delta_{m n} & \text { otherwise. }\end{cases}
$$


In addition, $S_{\Omega, \mathcal{M}}(\cdot)$ represents the Stokes-Coriolis Stratification to the corresponding linear equations of (1.2) using the Fourier transform under the assumption of $\mu=v$, which can be explicitly written as:

$$
S_{\Omega, \mathcal{M}}(t) u=\mathcal{F}^{-1}\left[\cos \left(\Omega \frac{|\eta|^{\prime}}{|\eta|} t\right) N_{1}+\sin \left(\Omega \frac{|\eta|^{\prime}}{|\eta|} t\right) N_{2}+N_{3}\right] *\left(e^{-\mu \Delta t} u\right)
$$

where

$$
|\eta|:=\sqrt{\eta_{1}^{2}+\eta_{2}^{2}+\eta_{3}^{2}},|\eta|^{\prime}:=\sqrt{\mathcal{M}^{2} \eta_{1}^{2}+\mathcal{M}^{2} \eta_{2}^{2}+\Omega^{2} \xi_{3}^{2}}
$$

and

$$
\begin{aligned}
& N_{1}=\left(\begin{array}{cccc}
\frac{\Omega^{2} \eta_{3}^{2}}{|\eta|^{\prime 2}} & 0 & -\frac{\mathcal{M}^{2} \eta_{1} \eta_{3}}{|\eta|^{\prime 2}} & \frac{\Omega \mathcal{M} \eta_{2} \eta_{3}}{|\eta|^{\prime 2}} \\
0 & \frac{\Omega^{2} \eta_{3}^{2}}{|\eta|^{\prime 2}} & -\frac{\mathcal{M}^{2} \eta_{2} \eta_{3}}{|\eta|^{\prime 2}} & -\frac{\Omega \mathcal{M} \eta_{1} \eta_{3}}{|\eta|^{\prime 2}} \\
-\frac{\Omega^{2} \eta_{1} \eta_{3}}{\mid \eta \eta^{\prime 2}} & -\frac{\Omega^{2} \eta_{2} \eta_{3}}{|\eta|^{\prime 2}} & \frac{\mathcal{M}^{2}\left(\xi_{1}^{2}+\xi_{2}^{2}\right)}{|\eta|^{\prime 2}} & 0 \\
\frac{\Omega \mathcal{M} \eta_{2} \eta_{3}}{|\eta|^{2}} & -\frac{\Omega \mathcal{M} \eta_{1} \eta_{3}}{|\eta|^{\prime 2}} & 0 & \frac{\mathcal{M}^{2}\left(\eta_{1}^{2}+\eta_{2}^{2}\right)}{|\eta|^{\prime 2}}
\end{array}\right),
\end{aligned}
$$

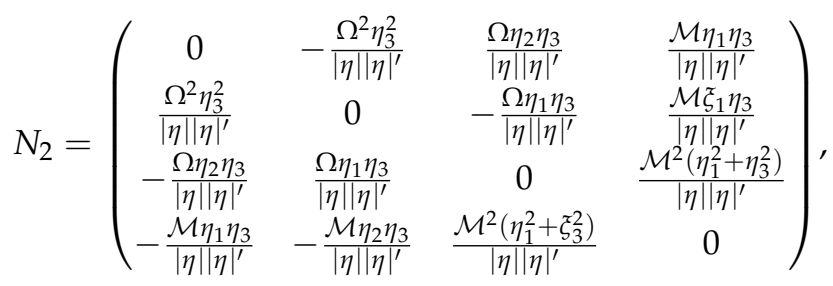

$$
\begin{aligned}
& N_{3}=\left(\begin{array}{cccc}
\frac{\mathcal{M}^{2} \eta_{3}^{2}}{|\eta|^{\prime 2}} & -\frac{\mathcal{M}^{2} \eta_{1} \eta_{2}}{|\eta|^{\prime 2}} & 0 & -\frac{\Omega \mathcal{M} \eta_{2} \eta_{3}}{|\eta|^{\prime 2}} \\
-\frac{\mathcal{M}^{2} \eta_{1} \eta_{2}}{|\eta|^{\prime 2}} & \frac{\mathcal{M}^{2} \eta_{1}^{2}}{|\eta|^{\prime 2}} & 0 & \frac{\Omega \mathcal{M} \eta_{1} \eta_{3}}{|\eta|^{\prime 2}} \\
0 & 0 & 0 & 0 \\
-\frac{\Omega \mathcal{M} \eta_{2} \eta_{3}}{|\eta|^{\prime 2}} & \frac{\Omega \mathcal{M} \eta_{1} \eta_{3}}{|\eta|^{\prime 2}} & 0 & \frac{\Omega^{2} \eta_{3}^{2}}{|\eta|^{\prime 2}}
\end{array}\right) .
\end{aligned}
$$

It is clear that a non-vanishing $N_{k l}^{j}$ satisfies the following condition, if we denote $(k, l)$-th element of matrix $N_{j}(\eta)$ by by $N_{k l}^{j}(\eta)$

$$
\left|N_{j k}^{l}\right| \leq 2 \text { for } \eta \in \mathbb{R}^{3}, j=1,2,3 \text { and } k, l=1,2,3,4 .
$$

In order to get the global well-posedness and analyticity of the viscous primitive equations of geophysics in critical variable exponent Fourier-Besov spaces, we use the following lemma on the existence of fixed point solutions.

Lemma 1 ([36]). Let $Y$ be a Banach space with norm $\|\cdot\|$ and $H: Y \times Y \rightarrow Y$ is a bounded linear operator that satisfies $\|H(a, b)\| \leq \varphi\|a\|\|b\|$ for all $a, b \in Y$ and a positive constant $\psi$. Then, the equation $y=x+H(a, b)$ has a solution $y \in Y$ such that $4 \varphi\|y\|<1$ for any $x \in Y$. Particularly, there is a solution such that $\|y\| \leq 2\|x\|$ and it is the only one such that $\|y\|<\frac{1}{2 \varphi}$.

Considering the integral form in Equation (6), we have to make a priori estimates to prove our main result. In the lemma given below, we prove the linear estimate for Equation (6).

Lemma 2. Let $\rho(\cdot), p_{1}(\cdot) \in \mathcal{P}\left(\mathbb{R}^{n}\right)$ for $p_{1}(\cdot) \leq \rho(\cdot), \beta(\cdot) \in C^{\log }\left(\mathbb{R}^{n}\right), 1 \leq \gamma, q \leq \infty$, $I=[0, T), T \in(0, \infty]$ and $u_{0} \in \mathcal{F} \dot{\mathcal{B}}_{\rho(\cdot), q}^{\beta(\cdot)+\frac{3}{\rho^{\prime}(\cdot)}}$, then

$$
\left\|S_{\Omega, \mathcal{M}}(t) u_{0}\right\|_{\mathcal{L}^{\gamma_{1}\left(I ; \mathcal{F} \mathcal{B}_{p_{1}(\cdot), q}^{\beta(\cdot)+}+\frac{3}{\rho^{\prime}(\cdot)}+\frac{2}{\gamma_{1}}\right)}} \lesssim\left\|u_{0}\right\|_{\mathcal{F} \dot{\mathcal{B}}_{\rho(\cdot), q}^{\beta(\cdot)+\frac{3}{\rho^{\prime}(\cdot)}}} .
$$


Proof. Since $S_{\Omega, \mathcal{M}}$ is a bounded Fourier multiplier, it can be simply estimated by an absolute constant. Let $\rho^{*}=\frac{\rho(y) p_{1}(y)}{\rho(y)-p_{1}(y)}$, by using Proposition 1 and considering $p_{1}(\cdot) \leq \rho(\cdot)$, we obtain

$$
\begin{aligned}
& \left\|S_{\Omega, \mathcal{M}}(t) u_{0}\right\|_{\left.\mathcal{L}^{\gamma_{1}\left(I ; \mathcal{F} \mathcal{B}_{p_{1}(\cdot), q}^{\beta(\cdot)+}\right.} \frac{3}{\rho^{3}(\cdot)}+\frac{2}{\gamma_{1}}\right)} \\
& =\|\| 2^{j\left(\beta(\cdot)+\frac{3}{\rho_{1}^{\prime}(\cdot)}+\frac{2 \beta}{\gamma_{1}}\right)} \varphi_{i} e^{-t|\cdot|^{2}} \widehat{u}_{0}\left\|_{\mathcal{L}^{\gamma_{1}\left(I ; L^{p_{1}(\cdot)}\right)}}\right\|_{l^{q}} \\
& \lesssim\left\|\sum_{k=0, \pm 1}\right\| 2^{j\left(\beta(\cdot)+\frac{3}{\rho^{\prime}(\cdot)}\right)} \varphi_{i} \widehat{u}_{0}\left\|_{L^{\rho(\cdot)}}\right\| 2^{j\left(\frac{3}{\rho_{1}^{\prime} \cdot(\cdot)}-\frac{3}{\rho^{\prime}(\cdot)}+\frac{2 \beta}{\gamma_{1}}\right)} \varphi_{j+k} e^{-t|\cdot|^{2}} \widehat{u}_{0}\left\|_{\mathcal{L}^{\gamma_{1}\left(I ; L^{\rho^{*}}\right)}}\right\|_{l^{q}} \\
& \lesssim\left\|\sum_{k=0, \pm 1}\right\| 2^{j\left(\beta(\cdot)+\frac{3}{\rho^{\prime}(\cdot)}\right)} \varphi_{j} \widehat{u}_{0}\left\|_{L^{\rho(\cdot)}}\right\| 2^{j \frac{2}{\gamma_{1}}} e^{-t 2^{2 \beta(j+k)}}\left\|_{\mathcal{L}^{\gamma_{1}(I)}}\right\| 2^{3 j\left(\frac{1}{\rho_{1}^{\prime}(\cdot)}-\frac{1}{\rho^{\prime}(\cdot)}\right)} \varphi_{j+k}\left\|_{L^{\rho^{*}}}\right\|_{l^{q}} \\
& \lesssim\left\|\sum_{k=0, \pm 1}\right\| 2^{3 j\left(\frac{1}{\rho_{1}^{\prime}(\cdot)}-\frac{1}{\rho^{\prime}(\cdot)}\right)} \varphi_{j+k}\left\|_{L^{\rho^{*}}}\right\| 2^{j\left(\beta(\cdot)+\frac{3}{\rho^{\prime}(\cdot)}\right)} \varphi_{j} \widehat{u}_{0}\left\|_{L^{\rho(\cdot)}}\right\|_{l q} \\
& \lesssim\|\| 2^{j\left(\beta(\cdot)+\frac{3}{\rho^{\prime}(\cdot)}\right)} \varphi_{j} \widehat{u}_{0}\left\|_{L^{\rho(\cdot)}}\right\|_{l^{q}} \\
& =\left\|u_{0}\right\|_{\mathcal{F} \dot{\mathcal{B}}_{p_{1}(\cdot), q}^{\beta(\cdot)+}}{\frac{3}{\rho^{\prime}(\cdot)}} \text {. }
\end{aligned}
$$

The following fact has been used in the above estimate:

$$
\begin{aligned}
& \sum_{k=0, \pm 1}\left\|2^{3 j\left(\frac{1}{\rho_{1}^{\prime}(\cdot)}-\frac{1}{\rho^{\prime}(\cdot)}\right)} \varphi_{j+k}\right\|_{L^{\rho^{*}}} \\
& =\sum_{k=0, \pm 1}\left\|2^{3 j\left(\frac{1}{\rho_{1}^{\prime}(\cdot)}-\frac{1}{\rho^{\prime}(\cdot)}\right)} \varphi_{j+k}\right\|_{L^{\frac{\rho(\cdot) p_{1}(\cdot)}{\rho(\cdot)-p_{1}(\cdot)}}} \\
& =\sum_{k=0, \pm 1} \inf \left\{\alpha>0: \int\left|\frac{2^{3 j\left(\frac{1}{\rho_{1}^{\prime}(\cdot)}-\frac{1}{\rho^{\prime}(\cdot)}\right)} \varphi_{j+k}}{\alpha}\right|^{\frac{\rho(\cdot) p_{1}(\cdot)}{\rho(\cdot)-p_{1}(\cdot)}} d x \leq 1\right\} \\
& =\sum_{k=0, \pm 1} \inf \left\{\alpha>0: \int\left|\frac{\varphi_{j+k}}{\alpha}\right|^{\frac{\rho(\cdot) p_{1}(\cdot)}{\rho(\cdot)-p_{1}(\cdot)}} 2^{-3 j} d x \leq 1\right\} \\
& =\sum_{k=0, \pm 1} \inf \left\{\alpha>0: \int\left|\frac{\varphi_{j+k}}{\alpha}\right|^{\frac{\rho\left(2^{j} \cdot\right) p_{1}\left(2^{j} \cdot\right)}{\rho\left(j^{\cdot} \cdot\right)-p_{1}\left(2^{j} \cdot\right)}} d x \leq 1\right\} \lesssim C .
\end{aligned}
$$

Theorem 1. Let $\rho(\cdot) \in C^{\log }\left(\mathbb{R}^{n}\right) \cap \mathcal{P}_{0}\left(\mathbb{R}^{n}\right), 2 \leq \rho(\cdot) \leq 6,1 \leq q<3$ and $\gamma \in[1, \infty]$. Then, there exists a positive constant $\varepsilon$ such that for any $u_{0} \in \mathcal{F} \dot{\mathcal{B}}_{\rho(\cdot), q}^{-1+\frac{3}{\rho^{3}(\cdot)}}\left(\mathbb{R}^{n}\right)$ that satisfies $\left\|u_{0}\right\|_{\mathcal{F} \dot{B}_{\rho(\cdot), q}{ }^{-1+} \frac{3}{\rho^{\prime}(\cdot)}\left(\mathbb{R}^{n}\right)}<\varepsilon$, Equation (1) has a unique global solution

$$
u \in \mathcal{L}^{\gamma}\left(\mathbb{R}_{+} ; \mathcal{F} \dot{\mathcal{B}}_{\rho(\cdot), q}^{-1+\frac{3}{\rho^{\prime}(\cdot)}+\frac{2}{\gamma}}\right) \cap \mathcal{L}^{\gamma}\left(\mathbb{R}_{+} ; \dot{\mathcal{B}}_{2, q}^{\frac{1}{2}+\frac{2}{\gamma}}\right) \cap \mathcal{L}^{\infty}\left(\mathbb{R}_{+} ; \dot{\mathcal{B}}_{2, q}^{\frac{1}{2}}\right)
$$

such that

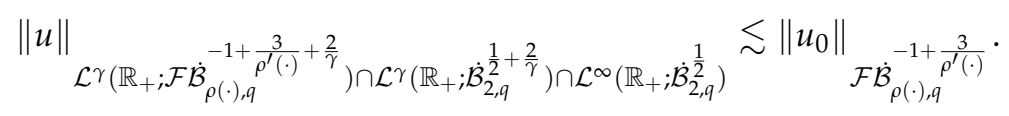


Proof. We define

$$
\mathcal{Z}:=\mathcal{L}^{\gamma}\left(\mathbb{R}_{+} ; \mathcal{F} \dot{\mathcal{B}}_{\rho(\cdot), q}^{-1+\frac{3}{\rho^{\prime}(\cdot)}+\frac{2}{\gamma}}\right) \cap \mathcal{L}^{\gamma}\left(\mathbb{R}_{+} ; \dot{\mathcal{B}}_{2, q}^{\frac{1}{2}+\frac{2}{\gamma}}\right) \cap \mathcal{L}^{\infty}\left(\mathbb{R}_{+} ; \dot{\mathcal{B}}_{2, q}^{\frac{1}{2}}\right)
$$

and we shall prove that the following is a contraction mapping.

$$
\Psi: u \rightarrow S_{\Omega, \mathcal{M}}(t) u_{0}+\int_{0}^{t} S_{\Omega, \mathcal{M}}(t-\mathcal{T}) \mathbb{P} \tilde{\nabla} \cdot(u \otimes u) d \mathcal{T}
$$

Next, we can write

$$
\begin{aligned}
\|\Psi\|_{\mathcal{Z}} & \leq\left\|S_{\Omega, \mathcal{M}}(t) u_{0}\right\|_{\mathcal{Z}}+\left\|\int_{0}^{t} S_{\Omega, \mathcal{M}}(t-\mathcal{T}) \mathbb{P} \tilde{\nabla} \cdot(u \otimes u) d \mathcal{T}\right\|_{\mathcal{Z}} \\
& =I_{1}+I_{2} .
\end{aligned}
$$

To estimate $I_{1}$, consider the hypothesis $\rho(\cdot) \geq 2$ and Lemma 2 , we obtain

$$
\begin{aligned}
& \left\|S_{\Omega, \mathcal{M}}(t) u_{0}\right\|_{\mathcal{L}^{\gamma}\left(\mathbb{R}_{+}, \mathcal{F} \dot{\mathcal{B}}_{\rho(\cdot), q}{ }^{\left.-1+\frac{3}{\rho^{\prime}(\cdot)}+\frac{2}{\gamma}\right)}\right.} \lesssim\left\|u_{0}\right\|_{\mathcal{F} \dot{\mathcal{B}}_{\rho(\cdot), q}{ }^{-1+\frac{3}{\rho^{\prime}(\cdot)}}}, \\
& \left\|S_{\Omega, \mathcal{M}}(t) u_{0}\right\|_{\mathcal{L}^{\gamma}\left(\mathbb{R}_{+}, \mathcal{F} \dot{\mathcal{B}}_{2, q}^{\left.\frac{1}{2}+\frac{2}{\gamma}\right)}\right.} \lesssim\left\|u_{0}\right\|_{\mathcal{F}_{\mathcal{B}(\cdot), q}{ }^{-1+\frac{3}{\rho^{\prime}(\cdot)}}}, \\
& \left\|S_{\Omega, \mathcal{M}}(t) u_{0}\right\|_{\mathcal{L}^{\infty}\left(\mathbb{R}_{+}, \mathcal{F} \dot{\mathcal{B}}_{2, q}^{\frac{1}{2}}\right)} \lesssim\left\|u_{0}\right\|_{\mathcal{F} \dot{\mathcal{B}}_{\rho(\cdot), q}{ }^{-1+\frac{3}{\rho^{\prime}(\cdot)}}},
\end{aligned}
$$

Hence, we obtain

$$
\left\|S_{\Omega, \mathcal{M}}(t) u_{0}\right\|_{\mathcal{Z}} \lesssim\left\|u_{0}\right\|_{\mathcal{F} \dot{\mathcal{B}}_{\rho(\cdot), q}-1+\frac{3}{\rho^{\prime}(\cdot)}}<\varepsilon
$$

To estimate $I_{2}$, let $\bar{\rho}(\cdot)=\frac{2 \rho(\cdot)}{2-\rho(\cdot)}$, using Propositions 1 and 2 , we have

$$
\begin{aligned}
& \left\|\int_{0}^{t} S_{\Omega, \mathcal{M}}(t-\mathcal{T}) \mathbb{P} \tilde{\nabla} \cdot(u \otimes u) d \mathcal{T}\right\|_{\mathcal{L} \gamma\left(\mathbb{R}_{+} ; \mathcal{F} \dot{\mathcal{B}}_{\rho(\cdot), q}{ }^{-1+}{\frac{3}{\rho^{\prime}(\cdot)}}^{\frac{3}{\gamma}}\right)} \\
& \left.\lesssim\|\| \int_{0}^{t} 2^{j\left(-1+\frac{3}{\rho^{\prime}(\cdot)}+\frac{2}{\gamma}\right)} \varphi_{j} e^{-(t-\mathcal{T})|\cdot|^{2}} \tilde{\nabla} \cdot \widehat{(u \otimes} u\right) d \mathcal{T}\left\|_{\mathcal{L}^{\gamma}\left(\mathbb{R}_{+} ; L^{\rho(\cdot)}\right)}\right\|_{l^{q}} \\
& \lesssim\|\| \int_{0}^{t}\left\|2^{j\left(\frac{3}{\rho^{\prime}(\cdot)}+\frac{2}{\gamma}\right)} \varphi_{j} e^{-(t-\mathcal{T})|\cdot|}\right\|_{L^{\bar{\rho}(\cdot)}}\left\|\Delta_{j}(u \otimes u)\right\|_{L^{6}} d \mathcal{T}\left\|_{\mathcal{L}^{\gamma}\left(\mathbb{R}_{+}\right)}\right\|_{l^{q}} \\
& \lesssim\|\| \int_{0}^{t} 2^{j\left(\frac{2}{\gamma}+\frac{5}{2}\right)} e^{-(t-\mathcal{T}) 2^{2 i}}\left\|2^{-3 j \frac{1}{\bar{\rho}(\cdot)}} \varphi_{j}\right\|_{L^{\bar{\rho}(\cdot)}}\left\|\Delta_{j}(u \otimes u)\right\|_{L^{6}} d \mathcal{T}\left\|_{\mathcal{L}^{\gamma}\left(\mathbb{R}_{+}\right)}\right\|_{l^{q}} \\
& \lesssim\|\| 2^{j\left(\frac{2}{\gamma}+\frac{1}{2}\right)}\left\|\Delta_{i}(u \otimes u)\right\|_{L^{\frac{6}{5}}}\left\|_{\mathcal{L}^{\gamma}\left(\mathbb{R}_{+}\right)}\right\| e^{-t 2^{2 j}} 2^{2 j}\left\|_{\mathcal{L}^{1}\left(\mathbb{R}_{+}\right)}\right\|_{l q} \\
& \lesssim\|u\|_{\mathcal{L} \gamma\left(\mathbb{R}_{+} ; \dot{\mathcal{B}}_{2, q}^{\frac{5}{2}+\frac{2}{\gamma}}\right)}\|u\|_{\mathcal{L}^{\infty}\left(\mathbb{R}_{+} ; L^{3}\right)} \\
& \lesssim\|u\|_{\mathcal{L}^{\gamma}\left(\mathbb{R}_{+} ; \dot{\mathcal{B}}_{2, q}^{\frac{1}{2}+\frac{2}{\gamma}}\right)}\|u\|_{\mathcal{L}^{\infty}\left(\mathbb{R}_{+} ; \dot{\mathcal{B}}_{2, q}^{\frac{1}{2}}\right)} .
\end{aligned}
$$

Likewise, we have

$$
\begin{aligned}
& \left\|\int_{0}^{t} S_{\Omega, \mathcal{M}}(t-\mathcal{T}) \mathbb{P} \tilde{\nabla} \cdot(u \otimes u) d \mathcal{T}\right\|_{\mathcal{L} \gamma\left(\mathbb{R}_{+} ; \mathcal{F} \dot{\mathcal{B}}_{\rho(\cdot), q}^{\frac{1}{2}+\frac{2}{\gamma}}\right)} \\
& \lesssim\|u\|_{\mathcal{L}^{\gamma}\left(\mathbb{R}_{+} ; \dot{\mathcal{B}}_{2, q}^{\frac{1}{2}+\frac{2}{\gamma}}\right)}\|u\|_{\mathcal{L}^{\infty}\left(\mathbb{R}_{+} ; \dot{\mathcal{B}}_{2, q}^{\frac{1}{2}}\right)^{\prime}}
\end{aligned}
$$


and

$$
\begin{aligned}
& \left\|\int_{0}^{t} S_{\Omega, \mathcal{M}}(t-\mathcal{T}) \mathbb{P} \tilde{\nabla} \cdot(u \otimes u) d \mathcal{T}\right\|_{\mathcal{L}^{\gamma}\left(\mathbb{R}_{+} ; \mathcal{F} \dot{\mathcal{B}}_{\rho(\cdot), q}^{\frac{1}{2}}\right)} \\
& \lesssim\|u\|_{\mathcal{L}^{\gamma}\left(\mathbb{R}_{+} ; \dot{\mathcal{B}}_{2, q}^{\frac{1}{2}+\frac{2}{\gamma}}\right)}\|u\|_{\mathcal{L}^{\infty}\left(\mathbb{R}_{+} ; \dot{\mathscr{B}}_{2, q}^{\frac{1}{2}}\right)} \cdot
\end{aligned}
$$

Hence, we obtain

$$
\left\|\int_{0}^{t} S_{\Omega, \mathcal{M}}(t-\mathcal{T}) \mathbb{P} \tilde{\nabla} \cdot(u \otimes u) d \mathcal{T}\right\|_{\mathcal{Z}} \lesssim\|u\|_{\mathcal{Z}}\|u\|_{\mathcal{Z}} .
$$

Using the inequalities (10) and (14) in (9), we can get

$$
\begin{aligned}
\|\Psi(u)\|_{\mathcal{Z}} & \leq C_{1}\left\|u_{0}\right\|_{\mathcal{F}^{-\dot{\mathcal{B}}}{ }^{-1+(\cdot), q} \frac{3}{\rho^{\prime}(\cdot)}}+C_{2}\|u\|_{\mathcal{Z}}\|u\|_{\mathcal{Z}} \\
& \leq C_{1}\left\|u_{0}\right\|_{\mathcal{F}_{\mathcal{B}}{ }^{-1+(\cdot), q} \frac{3}{\rho^{\prime}(\cdot)}}+C_{2} \varepsilon_{0}^{2} .
\end{aligned}
$$

Choosing $\varepsilon_{0}<\frac{1}{2 \max \left\{C_{1}, C_{2}\right\}}$ and for any $u_{0} \in \mathcal{F} \dot{\mathcal{B}}_{\rho(\cdot), q}^{-1+\frac{3}{\rho^{\prime}(\cdot)}}$ with

$$
\left\|u_{0}\right\|_{\mathcal{F} \dot{\mathcal{B}}_{\rho(\cdot), q}^{-1+\frac{\rho^{\prime}(\cdot)}{\rho^{\prime}}}}<\frac{\varepsilon_{0}}{2 \max \left\{C_{1}, C_{2}\right\}}
$$

we get

$$
\|\Psi(u)\|_{\mathcal{Z}} \leq \frac{\varepsilon_{0}}{2}+\frac{\varepsilon_{0}}{2}=\varepsilon_{0} .
$$

By applying Lemma 1, we can directly obtain that Equation (4) has a global unique solution with $\left\|u_{0}\right\|_{\mathcal{F} \dot{\mathcal{B}}_{\rho(\cdot), q}{ }^{-1+} \frac{3}{\rho^{\prime}(\cdot)}}<\varepsilon$ for sufficiently small $\varepsilon$.

\section{Gevrey Class Regularity}

Next, we prove Gevrey class regularity for the solution of Equation (4). The analyticity related to the NS equations has been studied by many authors. For more results in this direction, we refer $[37,38]$. The lemma given below is very important to get the spatial analyticity.

Lemma 3 ([39]). For all $x, y \in \mathbb{R}^{n}$ and $0<s \leq t<\infty$, the following inequality holds

$$
t|x|^{\frac{1}{2}}-\frac{1}{2}\left(t^{2}-s^{2}\right)|x|-s|x-y|^{\frac{1}{2}}-s|y|^{\frac{1}{2}} \leq \frac{1}{2} .
$$

Theorem 2. Let $\rho(\cdot) \in C^{\log }\left(\mathbb{R}^{n}\right) \cap \mathcal{P}_{0}\left(\mathbb{R}^{n}\right), 2 \leq \rho(\cdot) \leq 1,1 \leq q<3$, then, there exists a positive constant $\epsilon$ such that for any initial data $u_{0} \in \mathcal{F} \dot{\mathcal{B}}_{\rho(\cdot), q}^{-1+\frac{3}{\rho^{\prime}(\cdot)}}$ satisfying $\left\|u_{0}\right\|_{\mathcal{F H}_{\rho(\cdot), q}{ }^{-1+} \frac{3}{\rho^{\prime}(\cdot)}}<\epsilon$.

Equation (4) has an analytic unique solution $u$ in the sense that

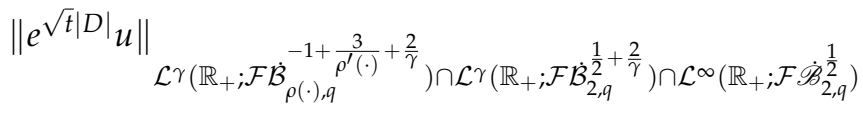

$$
\begin{aligned}
& \lesssim\left\|u_{0}\right\|_{\mathcal{F} \dot{\mathcal{B}}_{\rho(\cdot), q}-1+\frac{3}{\rho^{\prime}(\cdot)}} \cdot
\end{aligned}
$$


Proof. Assuming $g(t, y)=e^{\sqrt{t}|D|} u(t, y)$ and considering Equation (6), we get

$$
g(t, y)=e^{\sqrt{t}|D|} S_{\Omega, \mathcal{M}}(t) u_{0}+\int_{0}^{t} e^{\sqrt{t}|D|} S_{\Omega, \mathcal{M}}(t-\mathcal{T}) \mathbb{P} \tilde{\nabla} \cdot(u \otimes u) d \mathcal{T} .
$$

It is easy to obtain that

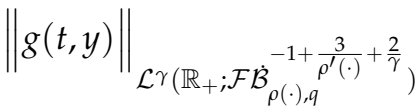

$$
\begin{aligned}
& \left.\leq\left\|e^{\sqrt{t}|D|} S_{\Omega, \mathcal{M}}(t) u_{0}\right\|_{\mathcal{L}^{\gamma}\left(\mathbb{R}_{+} ; \mathcal{F} \dot{\mathcal{B}} \rho(\cdot), q\right.}{ }^{-1+\frac{\rho^{\prime}(\cdot)}{}+\frac{2}{\gamma}}\right) \\
& +\left\|\int_{0}^{t} e^{\sqrt{t \mid}|D|} S_{\Omega, \mathcal{M}}(t-\mathcal{T}) \mathbb{P} \tilde{\nabla} \cdot(u \otimes u) d \mathcal{T}\right\|_{\left.\mathcal{L}^{\gamma\left(\mathbb{R}_{+} ; \mathcal{F} \dot{\mathcal{B}}_{\rho(\cdot), q}^{-1+} \frac{3}{\rho^{\prime}(\cdot)}+\frac{2}{\gamma}\right.}\right)} \\
& \lesssim\|\| e^{\sqrt{t}|\eta|-t|\eta|^{2}} 2^{i\left(-1+\frac{3}{\rho_{1}^{\prime}(\cdot)}+\frac{2}{\gamma}\right)} \varphi_{i} \widehat{u_{0}}\left\|_{\mathcal{L}^{\gamma}\left(\mathbb{R}_{+} ; L^{\rho(\cdot)}\right)}\right\|_{l^{q}} \\
& +\|\| 2^{i\left(\frac{3}{\rho^{\prime}(\cdot)}+\frac{2}{\gamma}\right)} \varphi_{i} \int_{0}^{t} e^{-\frac{1}{2}(t-\mathcal{T})|\eta|^{2}} \int_{\mathbb{R}^{n}} e^{\sqrt{t}|\eta|-\frac{1}{2}(t-\mathcal{T})|\eta|^{2}-\sqrt{\mathcal{T}}(|\eta-\zeta|+|\zeta|)} \\
& \left(\tilde { \nabla } \cdot \left(u(\eta-\widehat{\zeta, \mathcal{T})} \tilde{\nabla} P u(\zeta, \mathcal{T})) d \zeta d \mathcal{T}\left\|_{\mathcal{L}^{\gamma\left(\mathbb{R}_{+} ; L^{\rho(\cdot)}\right)}}\right\|_{l^{q^{*}}}\right.\right.
\end{aligned}
$$

Using $e^{\sqrt{t}|\eta|-\frac{1}{2} t|\eta|^{2}}=e^{\frac{1}{2}(\sqrt{t}|\eta|-1)^{2}+\frac{1}{2}} \leq e^{\frac{1}{2}}$ and by Lemma 3, we get

$$
\begin{aligned}
& \lesssim\|\| e^{-\frac{1}{2} t|\eta|^{2}} 2^{i\left(-1+\frac{3}{\rho^{\prime}(\cdot)}+\frac{2}{\gamma}\right)} \varphi_{i} \widehat{u_{0}}\left\|_{\mathcal{L}^{\gamma}\left(\mathbb{R}_{+} ; L^{\rho(\cdot)}\right)}\right\|_{l^{q}} \\
& +\|\| 2^{i\left(\frac{3}{\rho^{\prime}(\cdot)}+\frac{2}{\gamma}\right)} \varphi_{i} \int_{0}^{t} e^{-\frac{1}{2}(t-\mathcal{T})|\eta|^{2}}\left(\tilde { \nabla } \cdot \left(u(\eta-\widehat{\zeta, \mathcal{T})} \tilde{\nabla} P u(\zeta, \mathcal{T})) d \mathcal{T}\left\|_{\mathcal{L}^{\gamma}\left(\mathbb{R}_{+} ; L^{\rho(\cdot)}\right)}\right\|_{l^{q}} .\right.\right.
\end{aligned}
$$

The rest of the proof follows the same argument as Theorem 1, the details are therefore omitted.

\section{Conclusions}

We studied the primitive equations of geophysics in variable exponent Fourier-Besov spaces. Young's inequality is a significant tool to determine the global well-posedness of these kind of equations. Recently, Abbassi et al. [23] obtained the local well-posedness and global well-posedness results of Equation (4) for small initial data in Fourier-BesovMorrey spaces. In order to obtain the global well-posedness result, we cannot use Young's inequality in variable exponent function spaces. In this paper, we overcame this problem and obtained the global well-posedness results of Equation (4) for small initial data $a_{0}$ belonging to the homogeneous variable exponent in Fourier-Besov spaces. Furthermore, we proved the Gevrey class regularity of the solution.

Author Contributions: Conceptualization, M.Z.A. and N.U.; validation, N.U.; writing-original draft, M.Z.A. and N.U.; writing-review and editing, M.Z.A. and O.A.O.; funding acquisition, M.Z.A. and O.A.O. All authors have read and agreed to submit this version of the manuscript.

Funding: The research was supported by Zhejiang Normal University Postdoctoral Research fund under grant (No. ZC304020909, ZC304021907) and NSF of China (No. 10271437).

Acknowledgments: The authors are highly thankful to the editors and referees for their valuable comments and suggestions for the improvement of our manuscript.

Conflicts of Interest: The authors declare no conflict of interest. 


\section{References}

1. Cushman-Roisin, B.; Beckers, J.M. Introduction to Geophysical Fluid Dynamics: Physical and Numerical Aspects; Academic Press: Cambridge, MA, USA, 2011; Volume 101.

2. Pedlosky, J. Geophysical Fluid Dynamics; Springer Science \& Business Media: Berlin/Heidelberg, Germany, 2013.

3. Majda, A. Introduction to PDEs and Waves for the Atmosphere and Ocean; American Mathematical Soc.: Providence, RI, USA, 2003; Volume 9.

4. Babin, A.; Mahalov, A.; Nicolaenko, B. On the regularity of three-dimensional rotating Euler-Boussinesq equations. Math. Models Methods Appl. Sci. 1999, 9, 1089-1121. [CrossRef]

5. Cushman-Roisin, B. Introduction to Geophysical Fluid Dynamics; Prentice Hall: Englewood Cliffs, NJ, USA, 1994.

6. Leray, J. Sur le mouvement d'un liquide visqueux emplissant l'espace. Acta Math. 1934, 63, 193-248. [CrossRef]

7. Fujita, H.; Kato, T. On the nonstationary Navier Stokes initial value problem. Arch. Ration. Mech. Anal. 1964, 16, 269-315. [CrossRef]

8. Cannone, M.; Meyer, Y.; Planchon, F. Solutions auto-similaires des équations de Navier-Stokes. Semin. Equ. Aux Deriv. Partielles (Polytech.) 1994, 8, 1-10.

9. Koch, H.; Tataru, D. Well-posedness for the Navier-Stokes equations. Adv. Math. 2001, 157, 22-35. [CrossRef]

10. Lei, Z.; Lin, F.H. Global mild solutions of Navier-Stokes equations. Commun. Pure Appl. Math. 2011, 64, 1297-1304. [CrossRef]

11. Bourgain, J.; Pavlović, N. Ill-posedness of the Navier-Stokes equations in a critical space in 3D. J. Funct. Anal. 2008, 255, 2233-2247. [CrossRef]

12. Yoneda, T. Ill-posedness of the 3D Navier-Stokes equations in a generalized Besov space near BMO ${ }^{-1}$. J. Funct. Anal. 2010, 258, 3376-3387. [CrossRef]

13. Chemin, J.I.; Desjardins, B.; Gallagher, I.; Grenier, E. Anisotropy and dispersion in rotating fluids. In Nonlinear Partial Differential Equations and Their Applications: Collège de France Seminar; Elsevier: Amsterdam, The Netherlands, 2002; Volume XIV; pp. 171-191.

14. Giga, Y.; Inui, K.; Mahalov, A.; Saal, J. Uniform global solvability of the rotating Navier-Stokes equations for nondecaying initial data. Indiana Univ. Math. J. 2008, 57, 2775-2791. [CrossRef]

15. Hieber, M.; Shibata, Y. The Fujita-Kato approach to the Navier-Stokes equations in the rotational framework. Math. Z. 2010, 265, 481-491. [CrossRef]

16. Fang, D.; Han, B.; Hieber, M. Global Existence Results for the Navier-Stokes Equations in the Rotational Framework in Fourier-Besov Spaces. In Operator Semigroups Meet Complex Analysis, Harmonic Analysis and Mathematical Physics; Birkhäuser: Cham, Switzerland, 2015; pp. 199-211.

17. Konieczny, P.; Yoneda, T. On dispersive effect of the Coriolis force for the stationary Navier-Stokes equations. J. Differ. Equ. 2011, 250, 3859-3873. [CrossRef]

18. Abidin, M.Z.; Chen, J. Global well-posedness of the generalized rotating magnetohydrodynamics equations in variable exponent Fourier-Besov spaces. J. Appl. Anal. Comput. 2021, 11, 1177-1190. [CrossRef]

19. Chemin, J.Y. A propos d'un probleéme de pénalisation de type antisymétrique. J. Math. Pures Appl. 1997, 76, 739-755. [CrossRef]

20. Koba, H.; Mahalov, A.; Yoneda, T. Global well-posedness for the rotating Navier-Stokes-Boussinesq equations with stratification effects. Adv. Math. Sci. Appl. 2012, 22, 61.

21. Iwabuchi, T.; Mahalov, A.; Takada, R. Global solutions for the incompressible rotating stably stratified fluids. Math. Nachr. 2017, 290, 613-631. [CrossRef]

22. Charve, F.; Ngo, V.S. Global existence for the primitive equations with small anisotropic viscosity. Rev. Mat. Iberoam. 2011, 27, 1-38. [CrossRef]

23. Abbassi A.; Allalou C.; Oulha Y. Well-Posedness and Stability for the Viscous Primitive Equations of Geophysics in Critical Fourier-Besov-Morrey Spaces. In Nonlinear Analysis: Problems, Applications and Computational Methods. SM2A 2019; Hammouch Z., Dutta H., Melliani S., Ruzhansky M., Eds.; Lecture Notes in Networks and Systems; Springer: Cham, Switzerland, 2021; Volume 168

24. Orlicz, W. Über eine gewisse Klasse von Räumen vom Typus B. Bull. Int. Acad. Pol. Ser. A 1932, 8, $207-220$.

25. Musielak, J. Orlicz spaces. In Orlicz Spaces and Modular Spaces; Springer: Berlin/Heidelberg, Germany, $1983 ;$ pp. 33-111.

26. Nakano, H. Topology and Topological Linear Spaces; Maruzen Company: Tokyo, Japan, 1951.

27. Kovacik, O.; Rakosnik, J. On spaces $L^{p(x)}$ and $W^{k, p(x)}$. Czechoslov. Math. J. 1991, 41, 592-618.

28. Cruz-Uribe, D.; Diening, L.; Hästö, P. The maximal operator on weighted variable Lebesgue spaces. Fract. Calc. Appl. Anal. 2011, 14, 361-374. [CrossRef]

29. Cruz-Uribe, D.V.; Fiorenza, A. Variable Lebesgue Spaces: Foundations and Harmonic Analysis; Springer Science \& Business Media: Berlin/Heidelberg, Germany, 2013.

30. Abidin, M.Z.; Chen, J. Global well-posedness for fractional Navier-Stokes equations in variable exponent Fourier-Besov-Morrey spaces. Acta Math. Sci. 2021, 41, 164-176. [CrossRef]

31. Chen, Y.; Levine, S.; Rao, M. Variable exponent, linear growth functionals in image restoration. SIAM J. Appl. Math. 2006, 66, 1383-1406. [CrossRef]

32. Ruzicka, M. Electrorheological Fluids: Modeling and Mathematical Theory; Springer Science and Business Media: Berlin/Heidelberg, Germany, 2000. 
33. Fan, X. Global $C^{1, \alpha}$ regularity for variable exponent elliptic equations in divergence form. J. Differ. Equ. 2017, $235,397-417$. [CrossRef]

34. Almeida, A.; Hästö, P. Besov spaces with variable smoothness and integrability. J. Funct. Anal. 2010, 258, 1628-1655. [CrossRef]

35. Ru, S.; Abidin, M.Z. Global well-posedness of the incompressible fractional Navier-Stokes equations in Fourier-Besov spaces with variable exponents. Comput. Math. Appl. 2019, 77, 1082-1090. [CrossRef]

36. Bahouri, H.; Chemin, J.Y.; Danchin, R. Fourier Analysis and Nonlinear Partial Differential Equations; Springer Science \& Business Media: Berlin/Heidelberg, Germany, 2011; Volume 343.

37. Abidin, M.Z.; Chen, J. Global Well-Posedness and Analyticity of Generalized Porous Medium Equation in Fourier-Besov-Morrey Spaces with Variable Exponent. Mathematics 2021, 9, 498. [CrossRef]

38. Foias, C.; Temam, R. Gevrey class regularity for the solutions of the Navier-Stokes equations. J. Funct. Anal. 1989, 87, 359-369. [CrossRef]

39. Ferreira, L.C.; Villamizar-Roa, E.J. Exponentially-stable steady flow and asymptotic behavior for the magnetohydrodynamic equations. Commun. Math. Sci. 2011, 9, 499-516. [CrossRef] 